The Astronomical Journal, 116:2929-2935, 1998 December

(C) 1998. The American Astronomical Society. All rights reserved. Printed in U.S.A.

\title{
THE DISTANCE TO NGC 6397 BY M-SUBDWARF MAIN-SEQUENCE FITTING
}

\author{
I. NeILl REID \\ Palomar Observatory, 105-24, California Institute of Technology, Pasadena, CA 91125;inr@astro.caltech.edu \\ AND \\ JOHN E. GizIS \\ Department of Physics and Astronomy, LGRT 532A, University of Massachusetts, Amherst, MA 01003-4525; \\ gizis@stratford.phast.umass.edu \\ Received 1998 July 7; revised 1998 August 31
}

\begin{abstract}
Recent years have seen a substantial improvement both in photometry of low-luminosity stars in globular clusters and in modeling the stellar atmospheres of late-type dwarfs. We build on these observational and theoretical advances in undertaking the first determination of the distance to a globular cluster by main-sequence fitting using stars on the lower main sequence. The calibrating stars are extreme $M$ subdwarfs, as classified by Gizis, with parallaxes measured to a precision of better than $10 \%$. Matching against King et al.'s deep $[V,(V-I)]$ photometry of NGC 6397, and adopting $E_{B-V}=0.18$ mag, we derive a true distance modulus of $12.13 \pm 0.15 \mathrm{mag}$ for the cluster. This compares with $(m-M)_{0}=12.24 \pm 0.1$ derived through conventional main-sequence fitting in the $[V,(B-V)]$ plane. Allowing for intrinsic differences due to chemical composition, we derive a relative distance modulus of $\delta(m-M)_{0}=2.58 \mathrm{mag}$ between NGC 6397 and the fiducial metal-poor cluster M92. We extend this calibration to other metal-poor clusters and examine the resulting RR Lyrae $\left(M_{V},[\mathrm{Fe} / \mathrm{H}]\right)$ relation.

Key words: Galaxy: halo — globular clusters: individual (NGC 6397)
\end{abstract}

\section{INTRODUCTION}

The completion and publication of the results obtained by the Hipparcos astrometric satellite (ESA 1997) have led to a renaissance in classical and neoclassical astronomy. One of the latter disciplines is main-sequence fitting: estimating the distance to globular clusters based on matching their color-magnitude diagrams against a fiducial sequence defined by nearby subdwarfs of similar abundance and accurately known parallaxes (Sandage 1970). Several studies have applied Hipparcos data to this subject, with most (Reid 1997, 1998; Gratton et al. 1997; Chaboyer et al. 1998) concluding that the analysis favors larger distances, particularly for the extreme metal-poor systems, although Pont et al. (1998) recover the pre-Hipparcos results for M92. Combined with recent revisions in stellar models, however, even the last study leads to ages of less than 14 Gyr for the oldest clusters, while the longer distance-scale results point to ages of 11-13 Gyr.

Despite the addition of Hipparcos data, there are relatively few upper main-sequence (FG early-K) subdwarfs with parallaxes determined to an accuracy of better than $10 \%$. This reflects the scarcity of halo stars near the Sun: with a space density of $3 \times 10^{-6}$ stars $^{-1} M_{V}^{-1}$, one expects only 40-50 such stars within 100 pc. Not all of those subdwarfs have Hipparcos data, and many that were observed are sufficiently faint that parallaxes are measured to an accuracy of only 1.5-2 mas. In particular, the Hipparcos data set includes very few extreme subdwarfs $([\mathrm{Fe} / \mathrm{H}]<-1.5)$ with both reliable parallaxes and reliable abundance determinations.

By analogy with the disk, one expects the number density of halo subdwarfs to increase with decreasing mass, and surveys of both globular clusters (Fahlman et al. 1989; Paresce, Demarchi, \& Romaniello 1995) and the field (Dahn et al. 1995) show that this is indeed the case. Follow-up observations of proper-motion stars, particularly those drawn from the Luyten Half-Second catalog (Luyten 1980, hereafter LHS) have resulted in the identification of several dozen late-K and M-type subdwarfs within $50-100 \mathrm{pc}$ of the Sun. Most of those stars have sufficiently faint apparent magnitudes to permit ground-based CCD parallax measurements, which can achieve sub-milliarsecond precision (Monet et al. 1992). Moreover, with such faint apparent magnitudes, the transformation from relative to absolute parallaxes is robust.

In principle, M-subdwarfs could also be used as templates for main-sequence fitting distance determination. Until recently, there have been two substantial obstacles: first, globular cluster color-magnitude diagrams were defined poorly at $M_{V}>8$; second, reliable abundance estimates for late-type subdwarfs lay beyond the scope of stellar atmosphere models. The advent of WFPC2 on the Hubble Space Telescope (HST) has eliminated the first obstacle, while abundances in M-dwarfs can be at least constrained using the extensive theoretical analyses by Allard \& Hauschildt (1995).

Taking advantage of these advances, this paper presents the first attempt to use the main sequence defined by nearby extreme M-subdwarfs to estimate the distance of a metalpoor globular cluster. Section 2 outlines our calibration and selection of the appropriate local reference stars, and $\S 3$ matches those stars against the deep HST observations of NGC 6397 obtained by King et al. (1998a, hereafter $\mathrm{KACP}$ ). The final section summarizes the future prospects for this technique.

\section{THE SUBDWARF CALIBRATORS}

The existence of subluminous stars, lying between the disk main sequence and white dwarfs, was first suggested by Adams \& Joy (1922), who identified three weak-lined "Atype" stars (HD 19445, HD 219617, and HD 140283) with unusual absolute magnitudes, while the actual term subdwarf was coined by Kuiper (1939). Sandage \& Eggen (1959), however, were the first to demonstrate that these 
stars, subluminous in the observational $\left[M_{V},(B-V)\right]$ and $\left(M_{V}\right.$, spectral type) planes, also fell below the main sequence in $\left(M_{\text {bol }}, T_{\text {eff }}\right)$. Most early examples were drawn from proper motion surveys. Since the Galactic halo is a high-velocity dispersion, low rotation population, the typical subdwarf has a velocity of $200-250 \mathrm{~km} \mathrm{~s}^{-1}$ with respect to the Sun. As a result, while the local disk to halo number ratio is $\approx 400: 1$ by volume, the subdwarf contribution to catalogs of high proper-motion stars can exceed $25 \%$.

Initial studies concentrated on the more luminous $\mathrm{F}$ and G-type stars, although later-type high-velocity stars have been known for over 100 years-Kapteyn's star (G1 $\left.191-M_{V}=8.6\right)$ was identified in 1897. Later type (K and M) subdwarfs are characterized by strong metal hydride absorption, notably MgH (Greenstein 1971; Cottrell 1978) and CaH (Jones 1973; Mould 1976). Over 30 such stars now have accurate trigonometric parallax measurements, providing an empirical description of the lower main sequence in the HR diagram of metal-poor stars (Gizis 1997).

As one decreases the metal abundance of an M-type dwarf, TiO bands decrease in strength relative to metal hydride bands. This reflects partly the double-metal nature of TiO, partly the fact that titanium is in competition with hydrogen (via $\mathrm{H}_{2} \mathrm{O}$ ) for a decreasing supply of oxygen atoms (Mould 1976). Reid, Hawley, \& Gizis (1995) devised a series of narrowband indices to characterize the behavior of both $\mathrm{TiO}$ and $\mathrm{CaH}$, allowing subdwarfs to be ranked in terms of relative abundance. Gizis (1997) has used those indices to classify subdwarfs into two categories: intermediate (sdM) and extreme (esdM) subdwarfs (Fig. 1). One star stands out amongst the latter-LHS 453 , with CaH1 $~ 0.51$ but effectively no TiO absorption. The optical spectrum indicates that the abundance is substantially lower than that of the average esdM (Gizis 1997), but the position on the color-magnitude diagram $\left[M_{V}=13.08,(V-I)=2.22\right]$

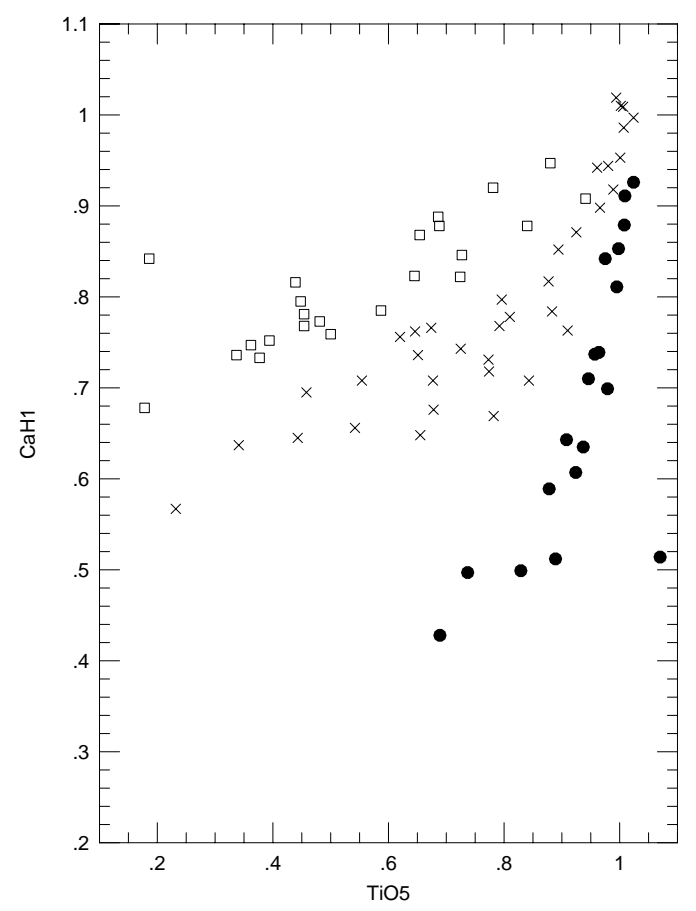

FIG. 1.-Identifying $M$ subdwarfs - the calibration of solar-abundance disk dwarfs (open squares), intermediate-abundance sdM subdwarfs (crosses), and extreme subdwarfs, esdM, in terms of the relative strength of $\mathrm{CaH}$ and $\mathrm{TiO}$ absorption. is not anomalous.

Transforming relative band strength measurements to metallicities requires appropriate model atmospheres. Gizis matches spectra spanning the wavelength range $\lambda \lambda 6200$ $7400 \AA$, including the $\mathrm{CaH}$ bands at $\lambda 6350$ and $\lambda 6800$, against models from the Extended grid calculated by Allard \& Hauschildt (1995). Based on that comparison, sdM subdwarfs appear to have abundances in the range -1 to -1.5 dex, while esdM subdwarfs fall in the range -1.5 to -2.5 dex, with an average abundance close to -2.0 dex. The latter stars should therefore be the local analogs of the $M$ subdwarfs found in the extreme halo clusters, such as NGC 6397, M15, and M92.

Support for this calibration comes from spectroscopy of low-luminosity common proper motion companions of three early-type subdwarfs of known abundance (Gizis \& Reid 1997b). In addition, one can compare the relative numbers of sdM and esdM stars against the $[M / \mathrm{H}]$ distribution derived by Laird, Carney, \& Latham (1988) for F and $G$ field subdwarfs. The latter distribution peaks at $[M / \mathrm{H}] \sim-1.5$, with a substantial tail to lower abundance, and one expects a similar distribution amongst lower luminosity stars. Clearly, the currently available sample of latetype subdwarfs with both accurate parallax measurements and spectroscopic observations is neither complete nor volume-limited, but since most attention has been devoted to stars lying well below the main sequence, one expects any bias to favor selection of the most metal-poor systems.

Following those arguments, we have identified the stars listed in Table 1 as local calibrators, defining a reference lower main sequence for the metal-poor $([M / \mathrm{H}]<-1.5)$ halo. All are spectroscopically confirmed esdM stars (Gizis \& Reid 1997a; Reid \& Gizis 1999, in preparation). Figure 2 plots the $\left[M_{V},(V-I)\right]$ color-magnitude diagram described by these stars, together with data for disk dwarfs drawn

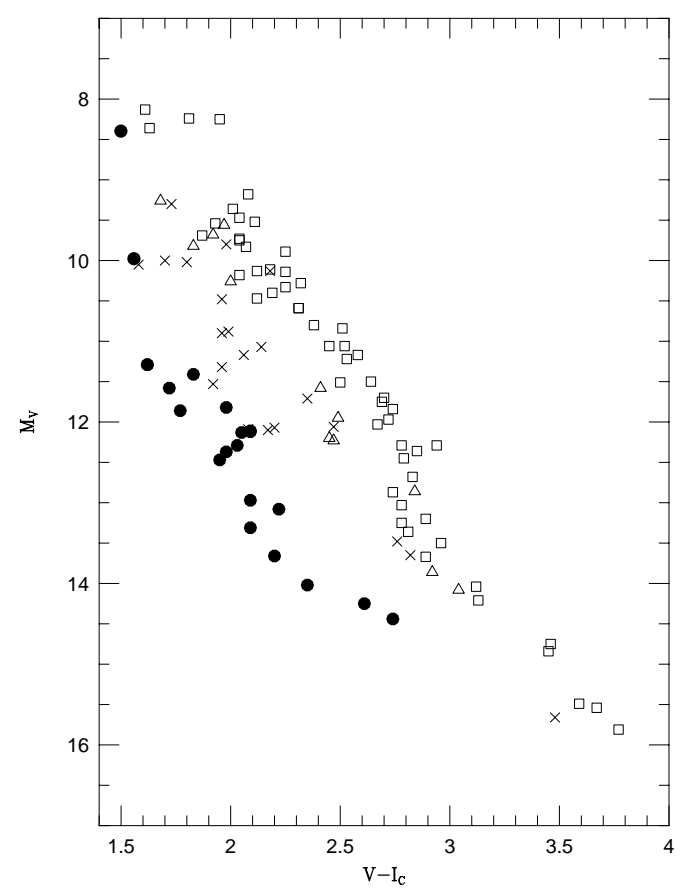

FIG. 2. $-\left[M_{V},(V-I)\right]$ color-magnitude diagram for late-type stars. Open squares identify single stars from the 8 pc sample (Reid \& Gizis 1997); open triangles are mildly metal-poor disk dwarfs; crosses are sdMs; and filled dots are esdM subdwarfs. 
TABLE 1

esdM Parallax SubdWarfs

\begin{tabular}{|c|c|c|c|c|c|c|c|}
\hline LHS & Name & $M_{V}$ & $(V-I)$ & Class & $\begin{array}{c}\pi \\
(\operatorname{arcsec})\end{array}$ & $\sigma_{\pi} / \pi$ & $\Delta L K$ \\
\hline 169 & Gl 129 & 11.58 & 1.72 & esdK 7 & 0.0309 & 0.074 & -0.06 \\
\hline 192 & LP $302-31$ & 12.37 & 1.98 & esdM1 & 0.0102 & 0.078 & -0.06 \\
\hline 364 & G165-47 & 12.47 & 1.95 & esdM1.5 & 0.0374 & 0.099 & -0.10 \\
\hline $375 \ldots$ & LP $857-48$ & 13.66 & 2.20 & esdM4 & 0.0395 & 0.025 & -0.01 \\
\hline $453 \ldots \ldots \ldots$ & LP139-13 & 13.08 & 2.22 & esdM3.5 & 0.0103 & 0.087 & -0.08 \\
\hline $522 \ldots$ & G1 861 & 11.29 & 1.62 & esdK 7 & 0.0268 & 0.078 & -0.06 \\
\hline $1174 \ldots \ldots$ & LP 406 & 12.97 & 2.09 & esdM3 & 0.0157 & 0.076 & -0.06 \\
\hline $1742 \mathrm{a} .$. & LP $417-42$ & 14.44 & 2.74 & esdM5.5 & 0.0134 & 0.090 & -0.08 \\
\hline $1970 \ldots \ldots$ & LP $484-6$ & 13.31 & 2.09 & esdM2.5 & 0.0129 & 0.062 & -0.04 \\
\hline $2045 \ldots$ & LP $545-45$ & 13.72 & 2.46 & esdM4.5 & 0.0111 & 0.081 & -0.07 \\
\hline $3061 \ldots \ldots$ & LP $502-32$ & 14.25 & 2.61 & esdM5 & 0.0089 & 0.090 & -0.08 \\
\hline $3382 \ldots \ldots \ldots$ & LP $24-219$ & 12.11 & 2.09 & esdM2.5 & 0.0104 & 0.087 & -0.08 \\
\hline $3390 \ldots \ldots$ & LP $181-51$ & 13.87 & 2.48 & esdM4.5 & 0.0123 & 0.081 & -0.07 \\
\hline $3548 \ldots \ldots \ldots$ & LP 695-96 & 12.12 & 2.09 & esdM3 & 0.0083 & 0.072 & -0.05 \\
\hline $3628 \ldots$ & LP $757-13$ & 12.12 & 2.05 & esdM1.5 & 0.0088 & 0.090 & -0.09 \\
\hline
\end{tabular}

Notes.-Parallax data from Monet et al. 1992 except:

LHS 169, 364, 522_-van Altena et al 1996;

LHS 375-Ruiz \& Anguita 1993.

from the 8 pc sample-the volume-limited sample of all stars north of $\delta=-30^{\circ}$, which are currently known to be within 8 pc of the Sun (Reid \& Gizis 1997).

Lutz-Kelker corrections (Lutz \& Kelker 1973) are usually invoked when combining trigonometric parallax data to derive statistical relations, such as determining a mean color-magnitude relation or in main-sequence fitting. The scale of those corrections depends both on the precision of the individual measurements, and on the distribution, $N(\pi)$, of the parent population. The latter can be regarded as the true density distribution as modified by parallaxindependent selection effects. Table 1 shows that most of the stars in our reference sample have parallaxes measured to a precision of better than $10 \%$, corresponding to systematic corrections of less than $0.1 \mathrm{mag}$. for a uniform space distribution. The latter corrections are listed as $\Delta L K$ in Table 1. However, since all of the calibrating subdwarfs were identified in surveys of high proper-motion stars, the underlying distribution $N(\pi)$ is far from uniform. One expects an initial rise as $\pi^{-4}$, but a subsequent flattening and turnover at $\pi_{\text {crit }}$, whose value depends on the proper motion limit and the subdwarf velocity distribution, and appropriate LK corrections will therefore be smaller than for $N(\pi) \propto \pi^{-4}$. To be conservative, we apply no Lutz-Kelker corrections in our main-sequence fitting analysis.

\section{THE DISTANCE TO NGC 6397}

NGC 6397 provides the best target for distance determination through main-sequence fitting using late-type subdwarfs. The cluster is one of the closest to the Sun, lying at Galactic coordinates $(l=338.2, b=-12.0)$, with an abundance of $[M / H]=-1.82$. (Carretta \& Gratton 1997). The latter metallicity estimate is based on spectroscopy of 10 red giant stars and is 0.34 dex higher than the same authors' calibration of M92, the fiducial extreme metalpoor cluster (Bolte \& Hogan 1995). Most importantly, NGC 6397 has deep imaging data obtained by the Hubble Space Telescope, extending to close to the hydrogenburning limit (Cool, Piotto, \& King 1996). The low latitude of NGC 6397 leads to substantial contamination of the color-magnitude diagram by field stars. However, the HST observations were made over a period of $3 \mathrm{yr}$, and that relatively short baseline, coupled with the high spatial resolution of the HST images and the significant transverse motion of NGC 6397, has allowed KACP to identify a proper-motion selected sample of cluster members, providing a color-magnitude diagram of unparalleled accuracy.

KACP's observations were made using the WFPC2 F555W and F814W filters, whereas our calibrating subdwarf observations are on the Cousins VI system. Fortunately, the two photometric systems have similar spectral response. Holtzman et al. (1995) provide analytic transformations between the natural WFPC system and the $U B V R I$ passbands, but those are limited to $(V-I)<1.5$. However, they also compute synthetic transformations by convolving the filter transmission curves with spectrophotometry of dwarfs, drawn from the Bruzual et al. spectral atlas, with $(V-I)$ colors as red $3.0 \mathrm{mag}$. As yet, there are no empirical tests of those predicted relations for $\mathbf{M}$ subdwarfs, but we are currently undertaking an HST snapshot program, searching for low-luminosity companions to such stars. Our observations are made using the F555W and F850LP filters, so we can test directly the transformations for the former, and indirectly assess the accuracy of the Holtzman et al. curves for $I$-band data.

To date, we have observations of six subdwarfs: LHS 169, $174,216,407,522$, and 320. All have ground-based $V, I$ data (Gizis 1997), with $(V-I)$ colors accurate to $\sim 0.05 \mathrm{mag}$. Comparing magnitudes derived from the HST F555W data, calibrated using STSDAS, against the ground-based data we derive $\delta V=\mathrm{F} 555 \mathrm{~W}-V=0.057 \pm 0.03 \mathrm{mag}$, where the uncertainty quoted is the standard deviation of residuals. Holtzman et al. predict a strong color term between the F850LP magnitudes and the standard Cousins $I$ band. Applying their specified calibration to derive $I_{850}$, we find $\delta I=I_{850}-I=0.012 \pm 0.06$. Given that some of the ground-based $I$-band data are transformed from $I_{K}$, this larger scatter is not unreasonable. These comparisons therefore suggest that $M$ subdwarfs do not exhibit pathological color terms in the transformation between the HST and ground-based broadband photometric systems. In the range $1.7<(V-I)<2.3$ spanned by the M92 stars, the color terms predicted by Holtzman et al. are almost parallel in $(V-\mathrm{F} 555 \mathrm{~W})$ and $(I-\mathrm{F} 814 \mathrm{~W})$. We have therefore taken 
the instrumental (F555W - I814W) colors as our best estimate of $(V-I)$, and adopt $V=\mathrm{F} 555 \mathrm{~W}-0.06 \mathrm{mag}$.

Accurate accounting of line-of-sight reddening is implicit in the derivation of the distance modulus, apparent or true, of a globular cluster by main-sequence fitting. Since NGC 6397 lies at low latitude, it is subject to significant foreground reddening, although previous estimates of this quantity are self-consistent. Most are based on the observed properties of blue horizontal branch stars: Graham \& Doremus (1968) set a lower limit of $E_{B-V} \geq 0.16$; Newell, Rodgers, \& Searle (1969) derived $E_{B-V}=0.20 \pm 0.02$; Cannon (1974) derives $E_{B-V}=0.18 \pm 0.02$, based on a direct comparison with BHB stars in NGC 6752; van den Bergh (1988) finds $E_{B-V}=0.19 \pm 0.02$; and Heber \& Kudritzki (1986) require $E_{B-V}=0.20$ in matching the ultraviolet spectrum of the sdO stars, ROB 162.

Other techniques of reddening estimation give similar results: Alcaino et al. (1997) estimated $E_{B-V}=0.17 \mathrm{mag}$, based on matching the $(U-B) /(B-V)$ distribution against data for NGC $1841\left(E_{B-V}=0.17\right)$; Cannon (1974) derives $E_{B-V}=0.18$ using the same technique, but calibrated against the Population I two-color relation; and Vandenberg, Bolte, \& Stetson (1990) match the mainsequence turnoff against M92 data to derive a differential reddening of $+0.17 \mathrm{mag}$, or $E_{B-V}=0.19$. Note that the last calculation does not take into account possible intrinsic color differences between the two clusters, a matter we return to in the following section. Finally, Schlegel, Finkbeiner, \& Davis (1998) have used IRAS and DIRBE data to derive extinction maps. Their estimated reddening in the direction of NGC 6397 is $E(B-V)=0.185 \pm 0.030$, in excellent agreement with other estimates.

Considering all of these results, we adopt a value of $E_{B-V}=0.18$ for the foreground reddening toward NGC 6397. Vandenberg et al. (1990) find evidence for some variation across the face of the cluster, but at a level of less than $0.015 \mathrm{mag}$. There is no evidence that the ratio of total to selective extinction, $R$, is enhanced (as is the case with M4), so we adopt $A_{V}=0.56 \mathrm{mag}$, and a ratio $E_{V-I} / E_{B-V}=1.35$ (Drukier et al. 1993; He et al. 1995), giving $E_{V-I}=0.24$. The dereddened color-magnitude diagram for the cluster is shown in Figure 3.

Pre-Hipparcos main-sequence fitting analyses indicated a true distance modulus for NGC 6397 of $(m-M)_{0}=$ 11.5-11.7 mag (Anthony-Twarog, Twarog, \& Suntzeff 1992), but those estimates were tied primarily to one refer-

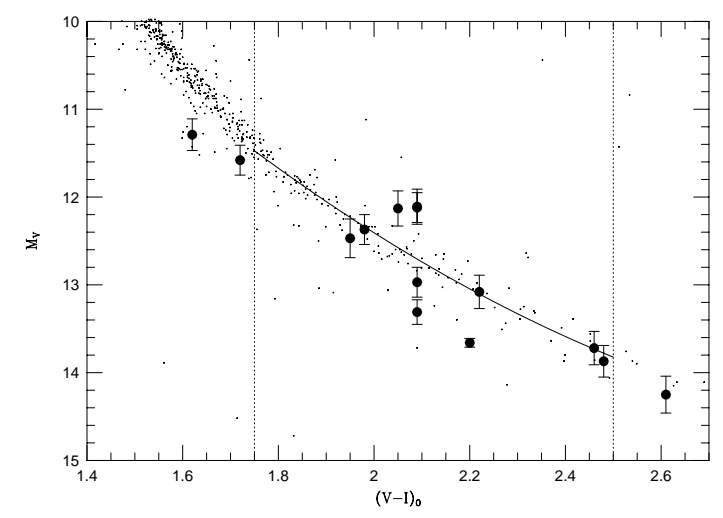

FIG. 3.-Main-sequence fitting to late-type subdwarfs in NGC 6397. The solid points are the calibrating esdMs, listed in Table 2; crosses mark data for proper-motion selected NGC 6397 members. The solid line plots the mean color-magnitude relation for the latter stars. ence star, HD 64090. The Hipparcos parallax for that star, $\pi=35.29 \pm 1.04$ mas, is $20 \%$ smaller than the previously accepted ground-based measurement, leading to an increase of at least $0.4 \mathrm{mag}$ in the inferred cluster distance modulus. Based on eight subdwarfs with $-1.55>[M / \mathrm{H}]>-2.1$ and with Hipparcos parallax measurements with a precision $\sigma_{\pi} / \pi<12 \%$, Reid (1998) derives $(m-M)_{0}=12.24 \pm 0.1$.

We have limited our current analysis to NGC 6397 stars in the color range $1.75 \leq V-I \leq 2.50$. This encompasses the majority of field esdMs but excludes both K subdwarfs, for which accurate metallicities are not available, and the few very low-luminosity esdMs, such as LHS 1742a. Even with the KACP proper motion data, some nonmembers appear in the cluster color-magnitude diagram (Fig. 3). We have identified the main-sequence locus by a "robust" linear fit to the KACP data with $V-I>1.5$. This minimizes the absolute value of the deviation of each point, rather than the square of deviation, and therefore gives little weight to outliers (Press et al. 1992). All objects within \pm 0.4 mag of the best-fit line are accepted. As can be seen in Figure 3, the main-sequence locus is recovered.

We then use least-squares fitting to describe the NGC 6397 main sequence in the range $1.75 \leq V-I \leq 2.50$. The best-fit third-order polynomial is

$$
V=24.517+3.436[(V-I)-2]-1.207[(V-I)-2]^{2} \text {. }
$$

As reference stars, we select the local esdM with parallaxes of precision better than $10 \%$, corresponding to uniform-density Lutz-Kelker corrections of less than 0.1 mag. If we fit a linear relation to the 10 esdM in Table 1 that meet this criterion, we derive

$$
M_{V}=12.429 \pm 0.076+(2.909 \pm 0.338)[(V-I)-2],
$$

which has a slope consistent with the NGC 6397 data and implies a distance modulus of $(m-M)_{0}=12.04$ at $V-I=2.0$. Alternatively, we can adopt the shape of the main-sequence defined by the NGC 6397 stars, and fit to the parallax stars, allowing only the zero point (evaluated at $V-I=2.0$ ) to vary. The resulting zero point is 12.403 , implying a true distance modulus of $12.13 \mathrm{mag}$. We have also fitted the cluster color-magnitude diagram with second- and fourth-order polynomials, and using the same technique, derive distance moduli of 12.12 and 12.14, respectively.

The main uncertainties in this distance determination center on the relative abundance of NGC 6397 and the mean of the esdM calibrators, and on how well-matched are the VI photometric systems. In the latter case, a systematic offset of $\delta(V-I)= \pm 0.03 \mathrm{mag}$ in the color transformation corresponds to $\pm 0.1 \mathrm{mag}$ in $\delta(m-M)$; in the former case, the metal-poor stellar models computed by Baraffe et al. (1997) indicate an offset of $\sim 0.3 \mathrm{mag}$ in $M_{V}$ between $[\mathrm{m} / \mathrm{H}]=-1.5$ and -2.0 at a color of $(V-I)=2.1 \mathrm{mag}$. Thus, a systematic error of \pm 0.25 dex in the mean abundance likely corresponds to $\pm 0.15 \mathrm{mag}$ in distance modulus.

Our estimate of the distance modulus of NGC 6397, derived by subdwarf-fitting to the lower main sequence, is $(m-M)_{0}=12.13 \pm 0.15 \mathrm{mag}$. Averaging this result with the $\left[M_{V},(B-V)\right]$ analysis, with double weight assigned to the latter result, gives $(m-M)_{0}=12.20$. The corresponding absolute magnitude at the turnoff is $M_{V}=3.8 \pm 0.1$, implying an age of $\sim 11 \mathrm{Gyr}$ if matched against either the models 
computed by D'Antona, Caloi, \& Mazzitelli (1997) or by Bergbusch \& Vandenberg (1992).

\section{THE DISTANCE TO M92}

M92 has been adopted generally as the fiducial cluster for the group of extreme halo globulars, including M15, M30, and M68, largely on account of its low foreground reddening. The standard estimate of $E_{B-V}=0.02 \mathrm{mag}$. is confirmed by Schlegel et al. (1998), who derive $0.022 \pm 0.003$. The available HST data for M92 do not extend to sufficiently faint magnitudes to allow a direct distance estimate using the techniques outlined above. However, we can use the methods outlined by Vandenberg et al. (1990) to derive a relative distance between M92 and NGC 6397.

The Vandenberg et al. study was aimed primarily at probing the relative ages of globular clusters, using the observed distribution in the color difference between the turnoff and the base of the giant branch in clusters of similar abundance. Aligning the upper main sequence and subgiant branch in clusters also gives a direct estimate of the difference in reddening, from $\Delta B-V$, and in apparent distance modulus, from $\Delta V$, provided one assumes that the intrinsic color and absolute magnitude at the turnoff are identical. Vandenberg et al. adopt the latter assumption in comparing M92 and NGC 6397, but that is not likely to be the case if the clusters have difference abundances. However, we can use theoretical stellar evolutionary tracks to account for the offset.

Carretta \& Gratton's (1997) red giant analyses indicate a metallicity difference of $\delta[\mathrm{Fe} / \mathrm{H}]=0.34$ dex (all differences are expressed in the sense M92-NGC 6397). King et al. (1998b, hereafter KSBD) derive a much lower abundance estimate for M92 (and, from Na I absorption, a reddening of $E_{B-V}=0.05-0.07$ ) based on high-resolution spectroscopy of subgiants. The high reddening is at odds with other studies, and there is no comparable abundance analysis of NGC 6397. Since we are concerned here with the relative metallicity of the two clusters, we adopt the Carretta \& Gratton result. Figure 3 of Vandenberg et al. shows that their color-difference age-calibration technique is relatively insensitive to metallicity at metallicities below $\sim-1.5$ dex. Thus, the general agreement between the M92 and NGC 6397 fiducial color-magnitude relations is consistent with there being no significant difference in age between the two clusters.

Given similar ages, we expect the higher abundance cluster, NGC 6397, to have a fainter, redder turnoff than M92. We can, however, use theoretical isochrones to allow explicitly for this effect in estimating the relative distance modulus. Defining $\delta(B-V)$ and $\delta M_{V}$ as the intrinsic offset in color and magnitude at the turnoff induced by the metallicity difference, the model calculations by D'Antona et al. (1997) predict values of

$$
\begin{gathered}
\delta(B-V)=-0.015 \mathrm{mag}, \\
\delta M_{V}=-0.07 \mathrm{mag}
\end{gathered}
$$

for the +0.34 dex NGC 6397/M92 abundance difference.

The offsets $\Delta(B-V)$ and $\delta V$, derived through the Vandenberg et al. technique of aligning cluster fiducial diagrams at the turnoff, can now be expressed in terms of differences in distance modulus, foreground reddening and in the intrinsic cluster properties, as follows,

$$
\begin{gathered}
\Delta(B-V)=\delta E_{B-V}+\delta(B-V), \\
\Delta V=\delta(m-M)_{0}-R \delta E_{B-V}-\delta M_{V} .
\end{gathered}
$$

Given measured values of $\Delta(B-V)=-0.17 \mathrm{mag}$ and $\Delta V=2.17$ mag for the M92/NGC 6397 pairing, adopting the standard value of $R=3.12$ leads to an estimate of $2.58 \mathrm{mag}$ for the difference in true distance modulus between M92 and NGC 6397. In $\S 3$ we derived an averaged value of $(m-M)_{0}=12.20 \pm 0.1$ for NGC 6397, giving $\left\langle(m-M)_{0}\right\rangle=14.78 \mathrm{mag}$ for M92, with an estimated uncertainty of $\pm 0.1 \mathrm{mag}$. Again, matching the turnoff absolute magnitude against either the D'Antona et al. or Bergbusch \& Vandenberg models leads to similar age estimates of 12-13 Gyr.

Comparing the current M92 distance modulus with other recent determinations, the Bolte \& Hogan (1995) semiempirical analysis, based on ground-based parallax data (notably for HD 103095), gives $(m-M)_{0}=14.65 \mathrm{mag}$, while D'Antona et al. (1997; echoed by Reid 1997) derive $(m-M)_{0}=14.80$ using a larger sample of reference stars. Of the post-Hipparcos analyses, Reid $(1997,1998)$ derives $(m-M)_{0}=14.94$, but the calibrating subdwarfs in the former analysis include binaries and are calibrated on the Carney et al. (1994) abundance scale, while the value cited in the latter paper uses Vandenberg et al.'s NGC 6397 $[\Delta V, \Delta(B-V)]$ method, but without correction for cluster to cluster metallicity differences. Gratton et al. (1997) find $(m-M)_{V}=14.82 \pm 0.08$ (for $E_{B-V}=0.03$ ), while the Chaboyer et al. (1998) distance modulus, based on eight calibrating subdwarfs, is $(m-M)_{0}=14.76$. Finally, Pont et al.'s result is $(m-M)_{0}=14.68$ if one excludes all potential binaries from their reference sample. Thus, the individual studies span a range of less than $0.15 \mathrm{mag}$ in true distance modulus. Moreover, as emphasized in the introduction, even the shortest distance estimate implies a cluster age of only $\sim 13$ Gyr, marking a significant easing of the cosmological constraints highlighted by Bolte $\&$ Hogan.

\section{THE RR LYRAE $\left(M_{V},[\mathrm{Fe} / \mathrm{H}]\right)$ RELATION}

Several recent studies have revisited the field RR Lyrae $\left(M_{V},[\mathrm{Fe} / \mathrm{H}]\right)$ calibration (Layden et al. 1996; Fernley et al. 1998; Gould \& Popowski 1998), and essentially reconfirmed the results originally derived by Strugnell, Reid, \& Murray (1986) and Barnes \& Hawley (1987): a mean absolute magnitude of $M_{V}=0.77 \pm 0.15$, independent of $[\mathrm{Fe} / \mathrm{H}]$. Gould \& Popowski and Udalski (1998) suggest that those results support a short distance scale $[(m-M)=18.1-18.2]$ to the LMC, and they argue for a systematic error in globular cluster distances derived by main-sequence fitting. NGC 6397 has no RR Lyrae population, but the instability strip is populated in the extreme metal-poor systems, notably M15. We briefly reexamine this issue based on the present results.

In the previous section we derived the reddening and distance modulus of M92 relative to NGC 6397, making due allowance for the abundance differences. Vandenberg et al. originally applied this same technique in estimating $[\Delta V$, $\Delta(B-V)]$ for a larger sample of cluster, choosing reference clusters of the appropriate metallicity. In particular, they calibrate M68 relative to M92 and M3 relative to NGC $6752\left[(m-M)_{0}=13.16, E_{B-V}=0.04\right.$; Reid 1998]. We have used the same method to estimate the relative distance 
TABLE 2

RR Lyrae Absolute Magnitudes

\begin{tabular}{cccccccccc}
\hline \hline Cluster & $E_{B-V}$ & {$[\mathrm{Fe} / \mathrm{H}]$} & $\Delta V$ & $\Delta(B-V)$ & Reference & $(m-M)_{0}$ & $\left\langle V_{0}\right\rangle(\mathrm{RR})$ & $M_{V}(\mathrm{RR})$ & RR Lyrae Data \\
\hline M5 $\ldots \ldots .$. & 0.02 & -1.11 & 0 & 0 & M5 & 14.50 & 15.00 & 0.50 & Reid 1996 \\
M3 ...... & 0.03 & -1.34 & -1.75 & 0.01 & NGC 6752 & 14.88 & 15.54 & 0.66 & Buonnano et al. 1994 \\
M68 ...... & 0.05 & -1.99 & -0.43 & -0.033 & M92 & 15.11 & 15.43 & 0.32 & Walker 1994 \\
M15 ..... & 0.09 & -2.12 & -0.57 & -0.07 & M92 & 15.23 & 15.55 & 0.32 & Silbermann \& Smith 1995 \\
M92..... & 0.02 & -2.16 & 0 & 0 & M92 & 14.78 & 15.04 & 0.26 & Carney et al. 1994 \\
\hline
\end{tabular}

NoTE.-Matching M3 against the upper main sequence of M13 leads to an inferred distance modulus of 15.10 mag.

modulus between M15 and M92. In addition, M5 has direct main-sequence fitting distance estimates by Gratton et al. (1997) and Reid (1998) based on Hipparcos subdwarfs, and we have averaged those to derive $(m-M)_{0}=14.50 .^{1}$ All of those clusters have substantial RR Lyrae populations, and Table 2 lists the corresponding mean absolute magnitude estimates for the variable star population.

The five clusters listed in Table 2 span a range of 1 dex in abundance and allow an estimate of the metallicity dependence of the absolute magnitudes of RR Lyraes. Those data are plotted in Figure 4 and compared with Layden et al.'s statistical parallax results; Sandage's $(1982,1993)$ cluster variable calibration, where the gradient $d M_{V} / d[\mathrm{Fe} / \mathrm{H}] \sim$ 0.3 is derived from period-shift analysis; Cacciari, Clementini, \& Fernley's (1992) and McNamara's (1997) field-star Baade-Wesselink analyses; and the relation predicted by Caloi, D'Antona, \& Mazzitelli's (1997) theoretical horizontal branch models (plotted from their Table 2 for $\log T_{\text {eff }}=$ 3.83). Cassisi et al. (1998) have recently computed OPALbased models that are in good agreement with the latter, favoring a slightly steeper $M_{V} /[\mathrm{Fe} / \mathrm{H}]$ relation. It is clear that the main-sequence fitting calibration is in close agreement with Sandage's analysis, McNamara's recent BaadeWesselink calibration and with Caloi et al.'s theoretical models.

Comparing specifically against the statistical parallax results, at intermediate abundances the discrepancy lies at the $1 \sigma$ level but is more significant at $[\mathrm{Fe} / \mathrm{H}]=-2$. Were we to adopt Gould \& Popowski's uniform calibration, $M_{V}(\mathrm{RR})=0.77$, the implied distance modulus of M92 is only $14.27 \mathrm{mag}$. This is $0.35-0.4 \mathrm{mag}$ lower than the shortest distance estimate (Pont et al. 1998) based on main-sequence fitting. Given the predominance of BHB stars in M92, evolutionary effects may lead to higher luminosities for that clusters RR Lyraes (Lee, Demarque, \& Zinn 1990), as suggested by the main-sequence fitting results, but, with wellpopulated instability strips, such considerations are not relevant to M15 and M68. Udalski does not discuss this issue; prompted partly by KSBD's results for M92, Gould $\&$ Popowski suggest that the origin may lie in a mismatch of the subdwarf and red giant cluster abundance scales. However, adjusting $(m-M)$ by $0.35 \mathrm{mag}$ in main-sequence fitting corresponds to a change of $-0.07 \mathrm{mag}$ in $(B-V)$ in the colors of the reference subdwarfs. In the case of M15 or M92, that adjustment implies an extremely low abundance. for example, the D'Antona et al. models predict $\partial(B-V) /$ $[\mathrm{Fe} / \mathrm{H}] \sim 0.05 \mathrm{mag} \mathrm{dex}^{-1}$ at $[\mathrm{Fe} / \mathrm{H}]=-2$, and that gradient should decrease with decreasing abundance and decreasing line-blanketing. Hence, a correction of -0.07

\footnotetext{
${ }^{1}$ As emphasized by Reid (1998), calibrating the distance to M5 based on $\mathrm{HD} 103095([\mathrm{Fe} / \mathrm{H}]=-1.22)$ alone gives $(m-M)_{0}=14.43$.
}

mag in $(B-V)$ is likely consistent with $\delta[\mathrm{Fe} / \mathrm{H}] \sim-2$, and an abundance of -4 dex for M92 seems extremely unlikely.

An alternative explanation, which accounts at least for the discrepancy at the lowest metallicities, may lie in the abundance distribution of the field star sample (Fig. 4). Only 22 stars in the Layden et al. sample have [Fe/ $\mathrm{H}]<-2$. On the other hand, the latter authors derive $\left\langle M_{V}\right\rangle=0.73 \pm 0.18$ for a sample of 83 variables with $\langle[\mathrm{Fe} /$ $\mathrm{H}]\rangle=-1.83$. Moreover, this proposal does not address the $0.2-0.3$ mag discrepancy at $[\mathrm{Fe} / \mathrm{H}] \sim-1$, emphasized by Reid (1998), between M5 and the Galactic Center variable stars. Thus, the conflict between the absolute magnitude calibrations defined by cluster and field RR Lyraes remains to be resolved.

\section{SUMMARY AND CONCLUSIONS}

We have used observations of nearby esdM subdwarfs to define the location of the lower main sequence of the metalpoor halo in the $\left[M_{V},(V-I)\right]$ color-magnitude diagram. Matching that calibrated sequence against deep HST pho-

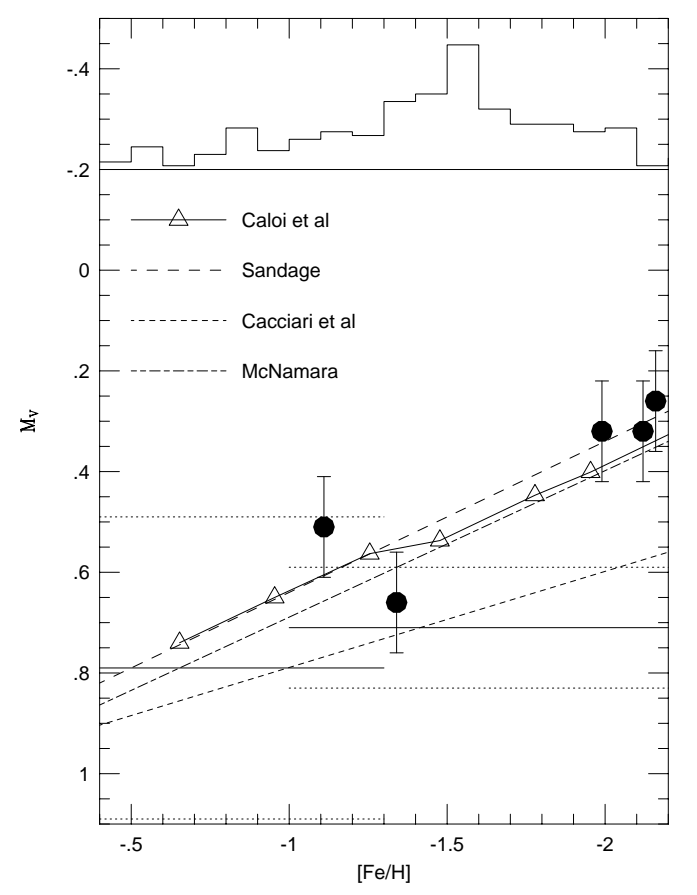

FIG. 4. $-\left(M_{V},[\mathrm{Fe} / \mathrm{H}]\right)$ relation for globular cluster RR Lyraes derived from the data listed in Table 2. The horizontal solid lines are the statistical parallax solution derived for field stars by Layden et al. (1996), with the dotted lines marking the $\pm 1 \sigma$ limits. Gould \& Popowski's field halo calibration is $M_{V}=0.77 \pm 0.15$. The linear relations plotted are from Cacciari et al.'s (1992) and McNamara's (1997) Baade-Wesselink field-star calibrations, and from Sandage's (1993) cluster variable calibration. The open triangles mark the predictions of the Caloi et al. (1997) zero-age horizontal branch models. The uppermost histogram plots the abundance distribution of the field RR Lyraes analyzed by Layden et al. 
tometry of NGC 6397, we estimate a cluster distance modulus of $12.12 \pm 0.15 \mathrm{mag}$ for a foreground reddening of $E_{B-V}=0.18$ mag. This is in excellent agreement with Reid's (1998) estimate of $(m-M)_{0}=12.24$, based on traditional main-sequence fitting in the $\left[M_{V},(B-V)\right]$ plane using earlier type subdwarfs with Hipparcos parallax data, and supports the larger distance of $\sim 2750 \pm 150 \mathrm{pc}$.

We have combined the predictions of theoretical evolutionary models with Vandenberg et al.'s measurements of relative offsets in the $[V,(B-V)]$ plane to estimate a distance to M92. Making allowance for the difference in abundance between the two clusters, we estimate M92 to lie at a distance modulus of $14.78 \pm 0.1 \mathrm{mag}$, a relatively modest increase over pre-Hipparcos determinations. Matched against the recent set of metal-poor stellar models computed by D'Antona et al. (1997), both clusters are estimated to have ages of $\sim 12 \pm 1$ Gyr. Extending the distance calibration to M15, M68 and M3, we derive absolute magnitudes for RR Lyrae variables that are in excellent agreement with both Sandage's $\left(M_{V},[\mathrm{Fe} / \mathrm{H}]\right)$ relation and the predictions of the recent horizontal branch models by Caloi et al. (1997).
The principal aim of this paper is to demonstrate that main-sequence fitting distance estimation need no longer be confined to using parallax stars on the upper main sequence. Deep, high spatial-resolution photometry with the Hubble Space Telescope already provides observations reaching the hydrogen-burning limit in the nearer globular clusters, while the most recent stellar atmosphere calculations allow reliable abundance estimates for the coolest subdwarfs. Further refinements in the models combined with observations with the future Next-Generation Space Telescope will allow the full exploitation of this latest variation on a neoclassical theme.

We would like to thank Messrs. King, Cool, Piotto, and Anderson for making available the full photometric catalog they have compiled of proper-motion members of NGC 6397. This work was supported partially by NASA grant GO-07385.01-96A from the Space Telescope Science Institute, which is operated by the Association of Universities for Research in Astronomy, Incorporated, under NASA contract NAS 5-26555.
Adams, W. S., \& Joy, A. H. 1922, ApJ, 56, 242

Alcaino, G., Liller, W., Alvarado, F., Kravtsov, V., Ipatov, A., Samus, N., \& Smirnov, O. 1997, AJ, 114, 1067

Allard, F., \& Hauschildt, P. H. 1995, ApJ, 445, 433

Anthony-Twarog, B. J., Twarog, B.,A., \& Suntzeff, N. B. 1992, AJ, 103, 1264

Baraffe, I., Chabrier, G., Allard, F., \& Hauschildt, P. H. 1997, A\&A, 327, 1054

Barnes, T. G., \& Hawley, S. L. 1987, ApJ, 307, L9

Bergbusch P. A., \& Vandenberg, D. A. 1992, ApJS, 81, 163

Bolte, M., \& Hogan, C. J. 1995, Nature, 376, 399

Buonnano, R., Corsi, C. E., Buzzoni, A., Caciari, C., Ferraro, F. R., \& Fusi Pecci, F. 1994, A\&A, 290, 69

Cacciari, C., Clementini, G., \& Fernley, J. A. 1992, ApJ, 396, 219

Caloi, V., D'Antona, F., \& Mazzitelli, I. 1997, A\&A, 320, 823

Cannon, R. D. 1974, MNRAS, 167, 551

Carney, B. W., Latham, D. W., Laird, J. B., \& Aguilar, L. A. 1994, AJ, 107, 2240

Carretta, E., \& Gratton, R. G. 1997, A\&AS, 121, 95

Cassisi, S., Castellani, V., Degl'Innocenti, S., \& Weiss, A. 1998, A\&AS, 129, 267

Chaboyer, B., Demarque, P., Kernan, P. J., \& Krauss, L. M. 1998, ApJ, 494, 96

Cool, A. M., Piotto, G., \& King, I. R. 1996, ApJ, 468, 655

Cottrell, P. L. 1978, ApJ, 223, 544

Dahn, C. C., Liebert, J., Harris, H. C., \& Guetter, H. H. 1995, in The Bottom of the Main Sequence - and beyond, ed. C. G. Tinney (Berlin: Springer), 239

D'Antona, F., Caloi, V., \& Mazzitelli, I. 1997, ApJ, 477, 519

Drukier, G. A., Fahlman, G. G., Richer, H. B., Searle, L., \& Thompson, I. 1993, AJ, 106, 2335

ESA. 1997, The Hipparcos Catalog (ESA SP-1200)

Fahlman, G. G., Richer, H. B., Searle, L., \& Thompson, I. B. 1989, ApJ, 343, L49

Fernley, J. A., Barnes, T. G., Skillen, I., Hawley, S. L., Hanley, C. J., Evans, D. W., Solano, E., \& Garrido, R. 1998, A\&A, 330, 515

Gizis, J. E. 1997, AJ, 113, 806

Gizis, J. E., \& Reid, I. N. 1997a, PASP, 109, 849 1997b, PASP, 109, 1233

Gould, A., \& Popowski, P. 1998, ApJ, 508, in press

Graham, J. A., \& Doremus, C. 1968, AJ, 73, 226

Gratton, R. G., Fusi Pecci, F., Carretta, E., Clementini, G., Corsi, C. E., \& Lattanzi, M. 1997, ApJ, 491, 749

Greenstein, J. E. 1971, in IAU Symp. 41, White Dwarfs, ed. W. J. Luyten, (Dordrecht: Reidel), 46

He, L. D., Whittet, D. C. B., Kilkenny, D., \& Jones, J. H. S. 1995, ApJS, 101, 335
Holtzman, J. A., Burrows, C. J., Casertano, C., Hester, J. J., Trauger, J. T., Watson, A. M., \& Worthey, G. 1995, PASP, 107, 1065

Jones, D. H. P. 1973, MNRAS, 161, 19P

King, I. R., Anderson, J., Cool, A. M., \& Piotto, G. 1998a, ApJ, 492, L37 (KACP)

King, J. R., Stephens, A., Boesgaard, A. M., \& Deliyaniis, C. P. 1998b, AJ, 115,666

Kuiper, G. P. 1939, ApJ, 89, 549

Laird, J. B., Carney, B. W., \& Latham, D. W. 1988, AJ, 95, 1843

Layden, A. C., Hanson, R. B., Hawley, S. L., Klemola, A. R., \& Hanley, C. J. 1996, AJ, 112, 2110

Lee, Y. W., Demarque, P., \& Zinn, R. 1990, ApJ, 350, 155

Lutz, T. E., \& Kelker, D. H. 1973, PASP, 85, 573

Luyten, W. J. 1980, The LHS Catalog (Minneapolis: Univ. Minnesota) (LHS)

McNamara, D. H. 1997, PASP, 109, 857

Monet, D. G., Dahn, C. C., Vrba, F. J., Harris, H. C., Pier, J. R., Luginbuhl, C. B., \& Ables, H. D. 1992, AJ, 103, 638

Mould, J. R. 1976, ApJ, 207, 535

Newell, E. B., Rodgers, A. W. \& Searle, L. 1969, ApJ, 156, 597

Paresce, F., Demarchi, G., \& Romaniello, M. 1995, ApJ, 440, 216

Pont, F., Mayor, M., Turon, C., \& Vandenberg, D. A. 1998, A\&A, 329, 87

Press, W. H., Teukolsky, S. A., Vetterling, W. T., \& Flannery, B. P. 1992

Numerical Recipes (2d ed.; (Cambridge: Cambridge Univ. Press)

Reid, I. N. 1996, MNRAS, 278, 367 1997, AJ, 114, 161

1998, AJ, 115, 161

Reid, I. N., \& Gizis, J. E. 1997, AJ, 113, 2246

Reid, I. N., Hawley, S. L., \& Gizis, J. E. 1995, AJ, 110, 1838

Ruiz, M. T., \& Anguita, C. 1993, AJ, 105, 614

Sandage, A. 1970, ApJ, 162, 841

.1982, ApJ, 252, 533 .1993, AJ, 106, 703

Sandage, A., \& Eggen, O. J. 1959, MNRAS, 119, 278

Schlegel, D. J., Finkbeiner, D. P., \& Davis, M. 1998, ApJ, 500, 525

Silbermann, N., \& Smith, H. A. 1995, AJ, 110, 704

Strugnell, P., Reid, I. N., \& Murray, C. A. 1986, MNRAS, 220, 413

Udalski, A. 1998, Acta. Astron., 48, 113

Van Altena, W. F., Lee, J. T., \& Hoffleit, E. D. 1996, The General Catalog of Trigonometric Parallaxes (New Haven: Yale Univ.)

van den Bergh, S. 1988, AJ, 95, 106

Vandenberg, D. A., Bolte, M., \& Stetson, P. B. 1990, AJ, 100, 445

Walker, A. 1994, AJ, 108, 555 